\title{
SUR LES RAYONS CATHODIQUES ;
}

\author{
Par M. P. VILLARD.
}

(Suite) (')

Émission. - Lorsqu'on diminue progressivement la pression dans un tube de Crookes, l'afflux cathodique, au début de son apparition, qui coïncide avec celle de l'espace obscur, couvre la cathode à peu près uniformément. Supposons d'abord celle-ci plane et centrée sur un tube d'un diamètre à peine supérieur au sien, cylindrique par exemple; si l'on continue à faire le vide, l'espace

(1) Voir p. 5 de ce rolume. 
obscur s'agrandit, l'afflux parcourt un trajet de plus en plus long, et l'action répulsive des parois intervient.

La partie extérieure du courant gazeux est repoussée vers les régions centrales; la densité du cylindre d'afflux augmente par suite à la périphérie, et le cercle d'impact sur la cathode présente alors une région centrale uniforme bordée d'un anneau plus lumineux. La photographie rend le phénomène très manifeste. A un vide plus avancé, les bords intérieurs de l'anneau se rejoignent, et l'uniformité se rétablit. Pendant toute cette évolution, la distance qui sépare le cylindre d'afflux de la paroi est, à une pression donnée, la même dans des tubes de divers diamètres, pourvu que l'anode soit éloignée. Ceci montre bien que le resserrement de l'afflux est dû à l'action des parois. La pression diminuant encore, le courant gazeux dont il s'agit se réduit peu à peu jusqu'à n'être plus qu'un filet de diamètre insensible et de moins en moins visible.

Le faisceau cathodique principal est, dans ces conditions, un cylindre plein, ayant pour base le cercle d'impact de l'afflux et présentant comme lui une condensation périphérique.

$\mathrm{Au}$ début de cette expérience, et tant que le cercle d'impact de l'afflux cathodique a un diamètre supérieur à 4 millimètres ou こ millimètres, la section affectée au passage de l'électricité, ou, si l'on veut, le débit électrique, sont suffisants pour que la différence de potentiel entre les électrodes soit faible et ne dépasse pas 15.000 à 20.000 volts, variant d'ailleurs fort peu avec la pression. Les rayons cathodiques sont nombreux, et leur énergie spécifique faible. A mesure que le cylindre d'afflux se resserre, une même diminution de diamètre réduit sa section dans une proportion croissante, et, quand ce diamètre n'est plus que de 1 millimètre environ, le passage de quelques centimètres cubes de mercure dans la trompe peut faire doubler la longueur de l'étincelle équivalente qui mesure la résistance du tube. L'énergie spécifique des rayons cathodiques augmente rapidement, et, à un certain moment, l'énergie totale du faisceau atteint un maximum se traduisant par une plus vive incandescence d'une lame de platine exposée au choc cathodique. Le maximum de production des rayons $\mathrm{X}$, lié probablement à ce qu'on pourrait appeler l'éclat cathodique, n'a généralement lieu que plus tard (').

(1) L'énergie spécifique nécessaire à l'obtention des rayons $\mathbf{X}$ peut être obtenue sans pousser le vide jusqu'à avoir un faisceau réduit: à un vide peu avancé. on 
Finalement l'afflux cathodique se réduit à zéro, l'émission cesse, et le courant électrique ne passe plus.

Ce qui précède est vrai d'une manière générale pour une cathode quelconque, les points d'émission étant seulement répartis différemment.

Si la cathode est fortement convexe, la convergence qu'elle imprime aux lignes de force est cause que l'afflux est plus dense à son centre qu'à sa périphérie; il en est de même du faisceau cathodique.

Le cas d'une cathode concave sphérique est plus intéressant, cette forme étant couramment employée.

Dès que l'espace obscur s'agrandit sensiblement, et que sa limite s'éloigne de l'électrode à une distance à peu près égale à son diamètre, l'afflux cathodique, tendant à arriver normalement à la surface de la cathode, en abandonne presque complètement la partie centrale. Attiré par le pourtour saillant de l'électrode et repoussé par les parois, il couvre seulement un anneau d'autant plus délié que la concavité est plus prononcée, et qui se resserre quand la pression diminue. On fait à volonté varier le diamètre de cet anneau en chargeant positivement une électrode auxiliaire passant dans un trou percé au centre de la cathode et dépassant celle-ci de 1 millimètre enviroñ.

L'émission cathodique est ainsi localisée presque complètement sur une zone, et le faisceau est un cône creux à parois plus ou moins épaisses. Ce phénomène, observé par M. Swinton( ${ }^{1}$ ), s'explique donc sans difficulté. La section radiante étant, toutes choses égales d'ailleurs, moindre qu'avec une cathode plane, la résistance est un peu plus forte.

A un degré de vide suffisant, variable, comme je l'ai montré, avec la forme et surtout le diamètre des tubes, le faisceau cathodique,

a souvent constaté que l'intercalation d'une étincelle dans le circuit suffit pour cela. L'explication de ce fait est simple : si, par exemple, l'étincelle éclate du côté cathode, celle-ci, primitivement presque au même potentiel que l'anode, est brusquement portée à un potentiel très différent. L'émission cathodique a lieu encore par les mêmes points; mais elle est moindre, et l'énergie spécifique des rayons augmente. Avec l'appareil Tesla, le résultat est encore plus net, et on peut arriver à supprimer complètement le faisceau central, la circulation gazeuse n'ayant pas le temps de s'établir; l'émission est très faible, et on obtient des rayons $\mathrm{X}$ à des pressions relativement considérables aussi bien qu'à un vide très avancé.

(1) Proceedings of the Royal Society of London, vol. LXI, n³70, p. 79. 
qu'il provienne d'une cathode plane, convexe ou concave, se réduit à un pinceau très fin. Celui-ci est toujours normal, au point de départ, à la surface d'émission. Ceci se vérifie aisément avec une cathode sphérique décentrée : le faisceau part du point de l'électrode situé

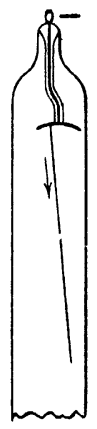

Fig. 1.

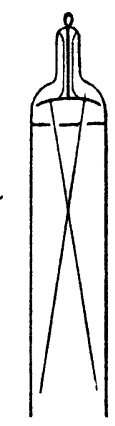

FIG. 2.

sur l'axe du tube et normalement à celle-ci $(f g .1)$. De même avec une cathode courbe, sphérique ou cylindrique, au-devant de laquelle, à 10 millimètres ou 10 milìmètres environ, on dispose un diaphragme présentant deux ou trois ouvertures qui délimitent autant de courants d'afflux, déterminant la production de faisceaux cathodiques correspondants ( $f g .2)$; les rayons émis s'infléchissent ensuite sous l'action répulsive de la cathode.

Propagation. - Deux faisceaux cathodiques sont sans action mutuelle appréciable. - Cette question, très controversée, paraît résolue par les expériences suivantes:

$1^{\circ}$ La disposition déjà décrite (4) d'une cathode plane, de 4 à こ centimètres de diamètre, précédée, à 10 ou 15 millimètres, d'un diaphragme métallique percé de deux ouvertures, permet d'avoir deux faisceaux cathodiques parallèles et étroits, passant par les centres des trous et se propageant dans un espace suffisamment protégé au point de vue électrique. On peut d'ailleurs, au moyen d'un large tube en toile métallique fixé au diaphragme, réaliser une véritable cage de Faraday. Dans ces conditions, les deux faisceaux cathodiques, même rapprochés à 8 millimètres l'un de l'autre, restent parallèles sur tout leur trajet, lequel est très visible si le

(1) Voir 9 p. de ce volume. 
vide est fait sur l'oxygène pur. L'emploi de la cage de Faraday ne change rien à ce résultat. On doit seulement prendre la précaution, si on rapproche les ouvertures, d'en réduire en même temps le diamètre. On évite ainsi que la cathode n’agisse obliquement, au travers de chacune d'elles, sur le faisceau qui a traversé l'autre. Entre le diaphragme et la cathode aucune action perturbatrice n'est à redouter, les lignes de force étant des droites.

En même temps on vérifie que deux courants d'affux cathodique ne se repoussent pas.

$2^{\circ} \mathrm{A}^{\mathrm{m}}, 30$ d'une cathode presque ponctuelle est un diaphragme à deux fentes rapprochées; à $0^{\text {n }}, 30$ au-delà est une lame fluorescente. L'ombre cathodique du système des deux fentes est égale à l'ombre géométrique, et les rayons passant par l'une des ouvertures ne changent pas de direction quand on ferme l'autre.

$3^{\circ}$ Un diaphragme à deux trous est placé en avant d'une cathode cylindrique concave ( $f g .2)$; on a ainsi deux faisceaux concourants, rectilignes à partir du diaphragme. Ces faisceaux se croisent sans s'influencer.

De même avec un tube à cinq cathodes concourantes placées dans des tubes distincts soudés à une ampoule unique, dans laquelle se fait le croisement. Le champ n'est très intense que près des cathodes, il est presque nul dans l'ampoule, et celle-ci constitue une sorte de cage de Faraday.

Il semble donc qu'il n'y ait pas lieu de parler d'action mutuelle s'exerçant lorsque deux faisceaux se pénètrent, ou que l'un d'eux rencontre des normales à l'autre cathode; ces conditions, considérées comme nécessaires et suffisantes par M. Deslandres ( ${ }^{(1)}$ et M. Goldstein $\left({ }^{2}\right)$, sont précisément réalisées dans la dernière des expériences précédentes, et aucune action ne se produit cependant. Dans la première expérience, au contraire (cathode plane précédée d'un diaphragme à deux trous), bien que le faisceau passant par l'une des ouvertures ne rencontre ni les rayons, ni les normales à la cathode qui traversent l'autre, une faible répulsion apparente aurait lieu, si on rapprochait beaucoup les deux trous sans réduire en même temps leur diamètre de manière à laisser entre eux un plein sulfisant. En pareil cas, et pour les raisons données plus haut, le diaphragme cesserait de constituer un écran efficace.

(1) Comptes Rendus, t. CXXIV, p. 681; 1897.

() Comples Renclus, t. CXXVI, p. 1199; 1898. 
Les rayons cathodiques ne sont déviés que quand ils coupent des lignes de force, et leur électrisation, découverte par M. Jean Perrin (1), suffit à expliquer les effets observés jusquà présent, si l’on tient compte en même temps de l'électrisation du tube. Dans l'expérience classique réalisée par Crookes avec un tube à deux cathodes placées en regard de deux fenêtres, chacune des cathodes repousse le faisceau émis par l'autre, et l'action subsiste évidemment, au moins près des cathodes, si on ferme l'une des fenêtres, comme l'ont fait MM. Wiedemann et Ebert. La disposition ingénieuse imaginée par M. Deslandres $\left({ }^{2}\right)$, et consistant à entourer par un tube de mica l'une des cathodes et le faisceau correspondant, fait disparaître la déviation; le mica se charge en effet comme les parois et fait écran; on a, en réalité, deux tubes de Crookes intérieurs l'un à l'autre, mais électriquement distincts.

Les phénomènes d'attraction et de répulsion observés par l'auteur précédent $\left({ }^{3}\right)$ paraissent également être la conséquence de l'état électrique du tube; ainsi une électrode placée au-devant et près de la cathode est, comme on l'a vu, à un potentiel beaucoup plus élevé que celle-ci et prend, par influence, une charge positive qui attire les rayons. Placée, au contraire, loin de la cathode, et par suite soumise presque uniquement à l'action des parois, elle se trouve dans un espace à potentiel très élevé presque uniforme. Si cette électrode présente une partie extérieure au tube ou, mieux, si elle est reliée à un conducteur extérieur, elle prend nécessairement une charge négative et repousse les rayons. L'action doit évidemment augmenter avec la capacité du conducteur, ce qui est conforme aux observations de M. Deslandres. Il y a également répulsion, pour les mêmes raisons, si l'électrode est reliée à la terre. Dans ce dernier cas surtout, la charge négative se manifeste par une émission de rayons cathodiques. Il suffit, d'ailleurs, de toucher un tube de Crookes avec le doigt, loin de la cathode, pour repousser le faisceau; mais il est facile de voir que la paroi touchée se charge négativement, car elle émet des rayons cathodiques et prend en même temps une teinte jaune (lumière du sodium), caractéristique de l'arrivée sur le verre d'un afflux cathodique.

Soit maintenant un tube à cathode plane centrée de même dia-

(1) Annales de Chimie et de Physique, 7º́rie, XI, p. 503.

() Loc. cit.

(3) Comptes Rendus, t. CXXIV, p. 680, 945, 1297 ; 1897.

J. de Phys., $3^{\circ}$ série, t. VIII. (Mars 1899.) 
mètre que lui à peu près. Les surfaces équipotentielles sont sensiblement planes, et le faisceau est cylindrique. Vient-on à réduire le diamètre de la cathode, les surfaces de niveau s'incurvent, et le faisceau est divergent. Si la cathode présente la forme d'un rectangle très allongé, les rayons cathodiques doivent s'étaler en éventail dans un plan perpendiculaire à la plus grande dimension du rectangle, plan dans lequel la courbure des surfaces équipotentielles est maxima. Il est facile de vérifier qu'il en est bien ainsi.

On voit que, pour obtenir un faisceau cathodique couvrant, à quelque distance, un espace notable, on devra employer une cathode très petite.
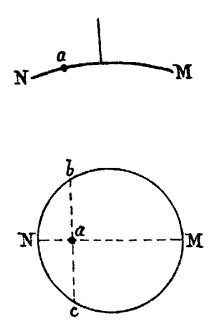

FIG. 3.

Supposons, au contraire, la cathode concave sphérique : à un vide peu avancé, les rayons émis forment un cône creux; l'un d'eux, partant d'un point $a(f g .3)$, est repoussé par la partie $b \mathbf{M} c$ de l'électrode plus fortement que par $b \mathrm{~N} c$; on peut encore dire que le projectile cathodique, dont ce rayon est la trajectoire, coupera obliquement les surfaces de niveau qu'il rencontrera successivement; il se comportera comme un corps pesant lancé obliquement de haut en bas; sa trajectoire s'infléchira, tendant à devenir parallèle à l'axe du cône; les génératrices de celui-ci seront donc curvilignes, tournant leur concavité vers l'extérieur, et il en résultera cet allongement bien connu du foyer cathodique, allongement d'autant plus marqué que le vide est plus avancé et le champ, par suite, plus intense près de la cathode. Plaçant au-devant de celle-ci un diaphragme à deux ouvertures ( $f g .2)$, le cône d'émission se réduit à deux faisceaux concourants, rectilignes à partir du diaphragme, se coupant cependant au-delà du centre de courbure de la cathode. Cette expérience montre que l'inflexion des trajectoires n'est pas due à une action 
mutuelle des rayons, puisqu'elle cesse au-delà du diaphragme et qu'elle se produit surtout au voisinage de la cathode, sur un trajet de 10 à 10 millimètres, là précisément où le champ présente son maximum d'intensité.

Diffusion des rayons cathodiques. - Lorsqu'un faisceau cathodique, suffisamment resserré déjà pour qu'il y ait production de rayons $\mathrm{X}$, rencontre la lame anticathodique d'un tube genre focus, une vive fluorescence du verre se produit dans toute la partie du tube située au-dessus du plan de l'anticathode ou d'un plan très voisin de celui-ci. Cette illumination hémisphérique a été attribuée par $\mathrm{M}$. Silvanus $\mathrm{P}$. Thomson à des rayons paracathodiques analogues aux rayons cathodiques, comme eux sensibles. à un champ magnétique ou électrostatique, mais incapables de produire des rayons $\mathrm{X}$. Leur point radiant est le point d'impact du faisceau direct.

Il s'agit, en réalité, de véritables rayons cathodiques aisément reconnaissables à leurs propriétés. On peut les considérer provisoirement comme provenant de la diffusion des rayons primaires, diffusion qui présente un maximum d'intensité dans une direction peu différente de celle qui correspond à la réflexion régulière.

$\mathrm{Si}$ on isole par un diaphragme un faisceau de ces rayons et qu'on le reçoive dans un tube latéral protégé électriquement, on peut s'assurer par le sens de la déviation magnétique ou électrique que ces rayons sont électrisés négativement.

En recevant ce faisceau sur une lame métallique, isolée ou reliée à l'anode, on obtient l'illumination hémisphérique du verre, comme dans le tube principal.

En mème temps il se produit des rayons X qu'il est facile de distinguer des rayons secondaires découverts par M. Sagnac et qui prennent naissance en même temps. L'approche d'un aimant, écartant de l'obstacle le faisceau en expérience sans agir sur les rayons $\mathrm{X}$ primaires qui l'accompagnent, fait effectivement cesser cette émission, au moins pour l'observation à l'écran fluorescent.

$\mathrm{Au}$ point de vue de la production des rayons $\mathrm{X}$, les rayons cathodiques diffusés se comportent vis-à-vis des obstacles rencontrés, verre ou métaux, exactement comme les rayons directs. On peut le constater en photographiant, à la chambre noire, une anticathode formée par la juxtaposition de diverses substances et que l'on expose : $1^{\circ}$ aux rayons cathodiques ordinaires; $2^{\circ}$ aux rayons diffusés. Un écran en aluminium ou en verre protège les plaques sensibles contre 
laction des rayons Sagnac. Avec des temps de pose convenablement choisis, les deux épreuves sont identiques.

Enfin les rayons diffusés pussèdent, au point de vue chimique, des propriètés identiques à celles des rayons directs. Il en sera question dans un autre article.

Il n'y a pas lieu de supposer que les rayons dontil s'agit sont émis directement par l'anticathode devenant cathode quand la décharge est oscillante. Le résultat est en effet le même, que l'anticathode soit anode ou isolée. En second lieu, quand il y a oscillation, l'émission par l'anode se fait suivant les lois relatives aux cathodes et produit les mêmes effets que l'inversion du courant par le commutateur de la bobine; en regard des deux faces de la lame anticathodique apparaissent, sur le verre, deux plages fluorescentes restreintes, et diamétralement opposées, présentant des irrégularités qui correspondent aux reliefs de la lame et au point d'attache du fil qui la porte. Ce phénomène, absolument distinct de l'illumination hémisphérique, à laquelle il se superpose en apparence quand il y a oscillation, cesse d'ailleurs si on fait diminuer la résistance du tube.

Il est permis d'admettre qu'au contact d'un obstacle les particules électrisées en mouvement, qui constituent les rayons cathodiques, se diffusent en tous sens, conservant, en partie au moins, leurs charges et leur énergie cinétique. De cette diffusion résultent de nouveaux rayons se propageant à peu près rectilignement, parce que le champ est très faible dans la région où ils se forment; à part leur mode particulier d'émission, ces rayons sont identiquesaux rayons directs.

J'ai précédemment montré que les rayons cathodiques, et aussi les rayons de Goldstein (Kanalstrahlen), se forment aux dépens d'un afflux de matière, chargée positivement, qui arrive à la cathode avec une vitesse considérable. Il est permis de supposer que cette matière est toujours la même, quel que soit le gaz sur lequel on ait fait le vide, quel que soit le métal des électrodes. Tous les tubes de Crookes, bien que préparés par des procédés variés, donnent les mêmes effets et présentent une allure unique. Les expériences de M. J.-J. Thomson (1) permettent d'être plus affirmatif encore: elles établissent en particulier que, pour une même chute de potentiel, la déviation magnétique des rayons est invariable, indépendante de la nature du

(1) Philosophical Magazine, 5゚ série, t. XLIV (oct. 1897); - Journal de Physique, t. VII, p. $39 ; 1898$. 
gaz sur lequel on a fait le vide. Sous ces divers rapports, les tubes de Crookes sont absolument différents des tubes de Geissler.

L'emploi du spectroscope fournit une première indication sur la

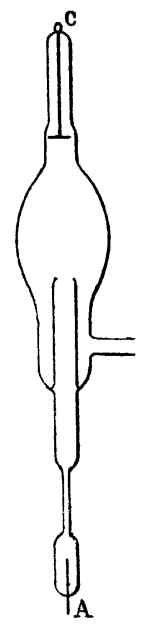

nature de la matière cathodique. L'instrument est dirigé vers la partie capillaire de l'appareil représenté $f g .1$, dans lequel on fait le vide sur l'air ou sur un gaz tel que l'oxygène, par exemple, qu il est facile de préparer pur et sec, en chauffant un peu d'oxyde de mercure dans un petit tube soudé à la trompe. Les vapeurs de mercure sont retenues par du sélénium; la vapeur d'eau est absorbée par de la potasse fondue au rouge. A un degré de vide variable avec la forme de l'appareil et ses dimensions, mais toujours tel qu'on ait un faisceau cathodique bien net et déjà étroit, on a, dans la partie capillaire, le spectre des gaz contenus dans le tube, plus celui de l'hydrogène. On observe alors, si $\mathrm{C}$ est cathode et $\mathrm{A}$ anode, que les raies de l'hydrogène s'affaiblissent rapidement et disparaissent complètement si l'expérience est faite avec soin. Le spectre des autres gaz reste invariable. Renversant le courant, les raies de l'hydrogène réapparaissent. Si on dirige, au contraire, le spectroscope vers la cathode et très près de celle-ci, on constate que, mème dans un tube bien sec, le spectre de l'hydrogène est remarquablement intense et parfois seul visible. Ainsi l'hydrogène semble jouer 
un rôle privilégié dans les phénomènes de Crookes et constituer le courant de matière qui circule dans les tubes à l'état radiant.

Cette première indication trouvée, il devient possible d'aborder la question par des procédés chimiques.

I. Rayons cathodiques. - Dans un tube de Crookes pouvant donner un faisceau un peu divergent, une lame de cuivre oxydé superficiellement est placée à $0^{\mathrm{m}}, 15$ environ en face de la cathode; entre cette lame et la cathode est interposé un petit obstacle en cuivre, oxydé également, fixé à la lame, et figurant par exemple une croix. L'ensemble peut être relié à l'anode, mais ceci importe peu. Le tube étant mis en activité, on observe, au bout de quelque temps, que l'ombre cathodique portée par la croix sur la lame se manifeste par une impression durable très nette, noire sur fond rouge. Examinant les choses de plus près, on constate que partout où le cuivre oxydé a été frappé par les rayons, il a pris une teinte rouge; les parties protégées, c'est-à-dire l'ombre de la croix, le revers de la lame et celui de l'obstacle, sont restées noires. Il est à supposer que les rayons cathodiques ont exercé sur l'oxyde une action réductrice. Le phénomène est parfaitement net, si l'on a soin d'éviter que la lame ne s'échauffe notablement, auquel cas l'oxyde noir serait décumposé et ramené à l'état de sous-oxyde rouge.

Il est préférable de prendre comme réactif un silicate. Par suite de la puissance de pénétration des rayons cathodiques, la réduction se fait alors dans la masse, à l'abri de l'atmosphère intérieure du tube.

Si, par exemple, la paroi anticathodique d'un tube de Crookes est recouverte intérieurement d'une mince couche de verre vert à l'oxyde cuivrique, ce revêtement se transforme en verre rouge cuivreux sous l'action des rayons cathodiques. De même le cristal est réduit : il noircit partout où il est frappé par les rayons cathodiques, comme au contact d'une flamme réductrice, à cela près que l'action est moins superficielle. Ceci explique la formation de taches brunes sur la paroi anticathodique de tubes de Crookes ayant beaucoup servi. Le verre ordinaire contient, en effet, presque toujours du plomb et brunit souvent au contact prolongé d'une flamme réductrice.

La réduction du cristal réussit, même si le vide est fait sur de l'oxygène très pur :

Un tube de Crookes, à cathode d'aluminium, présente une paroi anticathodique en cristal ; après lavage à l'acide nitrique bouillant, ce 
tube reçoit, par une ouverture qui sera scellée ensuite, un disque en cuivre profondément oxydé, dans lequel est pratiquée une découpure en forme de croix. Liappareil est alors relié à une trompe à mercure sans robinets, par l'intermédiaire de tubes contenant de l'oxyde de cuivre et de la potasse fondue au rouge. Une ampoule contenant un peu d'oxyde de mercure préalablement purifié par plusieurs chauffages est soudée à la canalisation qui, bien entendu, est toute en verre passé à la flamme. Après avoir fait le vide, on remplit l'appareil d'oxygène plusieurs fois sous une pression de quelques millimètres, et on le chauffe fortement chaque fois, en ayant soin d'insister particulièrement sur le tube de Crookes et l'oxyde de cuivre. Cette opération est renouvelée au cours des expériences. Dans ces conditions, on est sûr que l'atmosphère du tube est formée d'oxygène presque pur; d'ailleurs, le spectre de l'hydrogène n'est plus visible. Cependant l'action réductrice des rayons cathodiques n'est pas diminuée; ceux-ci, passant par la découpure pratiquée dans la lame de cuivre oxydé, vont marquer une croix noire sur le fond du tube. Le résultat est le même, si l'espace obscur est limité à quelques centimètres seulement de la cathode, et la réduction se fait alors dans une région où le spectre de l'oxygène est très brillant.

L'expérience peut encore se faire en recevant les rayons cathodiques sur une paroi de verre partiellement recouverte de cristal; ce dernier seul noircit, ce qui montre bien qu'il ne s'agit pas de parcelles arrachées aux électrodes.

La réduction du cristal réussit également dans un tube à éléctrodes extérieures, et on ne saurait invoquer ici la mise en liberté, par l'effet du courant, d'hydrogène ou d'oxyde de carbone condensés dans le métal.

Rayons cathodiques diffusés. - Les rayons cathodiques diffusés par les anticathodes possèdent, comme les rayons directs, la propriété de réduire le cristal même dans une atmosphère d'oxygène. Pour le constater, il suffit de faire fonctionner, pendant une heure ou deux, un tube dont l'anticathode est entourée d'un manchon de cristal, qu'il convient de prendre étroit, puisque, dans le cas présent, les rayons divergent en tous sens à partir d'un point. L'expérience terminée, on observe que le cristal est réduit dans toute la partie du manchon située au-dessus du plan de l'anticathode. La réduction n'est pas due aux rayons $\mathrm{X}$, car elle est empêchée par l'interposition d'une mince feuille d'aluminium $\left(0^{\mathrm{mm}}, \mathbf{1}\right)$. 
Afflux cathodique. - Si la.partie centrale d'une cathode plane est remplacée par une lame de cristal, ce qui ne modifie pas sensiblement la marche de l'afflux ni la production du faisceau cathodique central, on constate que le cristal est profondément réduit là où il est frappé par le courant gazeux, et le cercle d'impact de l'afflux marque ainsi sa trace sur la lame. Cette trace se réduit presque à un point central dans un tube de révolution, quand le vide est suffisamment poussé. Cette expérience peut être combinée avec l'une des précédentes et on obtient ainsi une réduction aux deux extrémités du faisceau cathodique.

Rayons de Goldstein. - Une lame de cristal, placée sur le trajet des rayons de Goldstein, est rapidement réduite. Ce résultat pouvait être prévu, les rayons dont il s'agit n'étant autre chose que le prolongement de l'afflux cathodique, qui continue sa route au travers d'une cathode perforée, après avoir perdu sa charge.

Ainsi les rayons cathodiques, les rayons de Goldstein, et l'afflux cathodique paraissent formés aux dépens d'une matière possédant d'une manière constante la propriété de réduire certains oxydes, et cela indépendamment de son état électrique, qui est l'état neutre dans le cas des rayons de Goldstein.

Le résultat est le même, soit qu'on opère sans précautions spéciales, soit au contraire qu'on fasse le vide sur de l'oxygène très pur, ou même qu'on supprime les électrodes intérieures. Il semble donc que l'on chercherait vainement à obtenir des rayons cathodiques formés aux dépens d'une matière quelconque.

Or le seul gaz réducteur que l'on puisse trouver dans un tube sans électrodes, lavé, puis chauffé fortement, est évidemment l'hydrogène ; c'est précisément celui que le spectroscope montre se portant à la cathode à l'exclusion des autres. Son origine est facile à trouver ; on ne saurait, en effet, se débarrasser complètement de l'eau par des desséchants, et le verre peut en fournir presque indéfiniment, surtout si on le chauffe; la régénération des tubes de Crookes par un séjour suffisant à l'étuve se trouve ainsi expliquée sans peine.

J'ajouterai qu'avec un tube à électrodes de mercure, dans lequel le vide a été fait avec beaucoup de soin sur le mercure bouillant, je n'ai observé que le phénomène de Geissler; il ne s'est pas formé de rayons cathodiques.

Les propriétés physiques et chimiques de l'hydrogène font déjà de ce gaz un corps à part dans la série des éléments; il n'est nulle- 
ment invraisemblable d'admettre qu'il possède, et cela exclusivement, la propriété de pouvoir donner des rayons cathodiques. 